University of Wollongong

Research Online

Faculty of Engineering and Information

Faculty of Engineering and Information

Sciences - Papers: Part A

Sciences

$1-1-2020$

\title{
Automatic ventricular nuclear magnetic resonance image processing with deep learning
}

Binbin Yong

Chen Wang

Jun Shen

University of Wollongong, jshen@uow.edu.au

Fucun Li

University of Wollongong, f1626@uowmail.edu.au

Hang Yin

Follow this and additional works at: https://ro.uow.edu.au/eispapers

Part of the Engineering Commons, and the Science and Technology Studies Commons

Research Online is the open access institutional repository for the University of Wollongong. For further information contact the UOW Library: research-pubs@uow.edu.au 


\title{
Automatic ventricular nuclear magnetic resonance image processing with deep learning
}

\begin{abstract}
Cardiovascular diseases (CVD) seriously threaten the health of human beings, and they have caused widespread concern in recent years. At present, the diagnosis of CVD is mainly conducted by computed tomography (CT), echocardiography and nuclear magnetic resonance (NMR) technologies. NMR imaging technology is widely used in medical applications owing to its characteristics of high resolution and very low radiation. However, manual NMR image segmentation is time-consuming and error-prone, which has led to the research on automatic NMR image segmentation technologies. Researchers tend to explore the ventricular NRM image segmentation to improve the accuracy of CVD diagnosis. In this study, based on deep learning technology, we propose a layered Mask R-CNN segmentation method to segment ventricular NMR images. The experimental results show that the mean dice metrics (DM) of left ventricular segmentation and right ventricular segmentation are 0.92 and 0.89 , and the Hausdorff distance (HD) metrics are $4.78 \mathrm{~mm}$ and $7.03 \mathrm{~mm}$. Our research indicates that the proposed novel method has great potential to automate the ventricular NMR image segmentation. We also discuss the automatic abnormal ventricular systolic function detection method based on the proposed layered segmentation model.
\end{abstract}

\section{Keywords}

image, resonance, magnetic, deep, nuclear, processing, ventricular, automatic, learning

\author{
Disciplines \\ Engineering | Science and Technology Studies
}

\section{Publication Details}

Yong, B., Wang, C., Shen, J., Li, F. \& Yin, H. (2020). Automatic ventricular nuclear magnetic resonance image processing with deep learning. Multimedia Tools and Applications, Online first 1-17. 


\title{
Automatic ventricular nuclear magnetic resonance image processing with deep learning
}

\author{
Binbin Yong · Chen Wang · Jun Shen • \\ Fucun Li • Hang Yin • Rui Zhou*
}

Received: date / Accepted: date

\begin{abstract}
Cardiovascular diseases (CVD) seriously threaten the health of human beings, and they have caused widespread concern in recent years. At present, the diagnosis of CVD is mainly conducted by computed tomography (CT), echocardiography and nuclear magnetic resonance (NMR) technologies. NMR imaging technology is widely used in medical applications owing to its characteristics of high resolution and very low radiation. However, manual NMR image segmentation is time-consuming and error-prone, which has led to the research on automatic NMR image segmentation technologies. Researchers tend to explore the ventricular NRM image segmentation to improve the accuracy of CVD diagnosis. In this study, based on deep learning technology, we propose a layered Mask R-CNN segmentation method to segment ventricular NMR images. The experimental results show that the mean dice metrics $(\mathrm{DM})$ of left ventricular segmentation and right ventricular segmentation are 0.92 and 0.89 , and the Hausdorff distance (HD) metrics are $4.78 \mathrm{~mm}$ and 7.03 $\mathrm{mm}$. Our research indicates that the proposed novel method has great potential to automate the ventricular NMR image segmentation. We also discuss the automatic abnormal ventricular systolic function detection method based on the proposed layered segmentation model.
\end{abstract}

Keywords Cardiovascular disease · NMR image segmentation · layered Mask R-CNN · ventricular systolic function detection

\footnotetext{
* Corresponding Author

Binbin Yong · Chen Wang · Hang Yin · Rui Zhou

School of Information Science \& Engineering, Lanzhou University, Lanzhou, Gansu, China Tel.: +86-931-8912025

Fax: +86-931-8912025

E-mail: \{yongbb, chenwang15, yinh2016,zr\}@lzu.edu.cn

Jun Shen · Fucun Li

School of Computing and Information Technology, University of Wollongong, NSW, Australia, 2522

E-mail: jshen@uow.edu.au,fl626@uowmail.edu.au
} 


\section{Introduction}

Nowadays, cardiovascular disease (CVD) has been a great threat to human health $[4,17]$. There are more than 10 million people dying of CVD annually all over the world. With the rapid development of medical technology, CVD can be diagnosed by medical images and various indicators. Nuclear magnetic resonance (NMR) imaging technology can be used in ventricular detection to generate arbitrary angle image sequences of the heart, which can be used to observe heart health. At present, the ventricular areas need to be manually segmented before they are used for CVD diagnosis by professional doctors. In recent years, clinical practice shows that many CVD diagnoses need to be judged by interpreting NMR segmentation images. However, traditional ventricular image segmentation methods are time-consuming, laborious and errorprone, which undoubtedly leads to the information loss or even misdiagnosis. Indeed, many traditional methods can not meet the accuracy requirement of ventricular NMR image segmentation, and the application scenarios are limited. At present, many NMR machines provide automatic segmentation function. However, because of the poor effectiveness of segmentation algorithm, these machines are inaccurate and inefficient. Therefore, the research for an efficient, accurate automatic ventricular NMR image segmentation method has far-reaching significance for accurate medical diagnosis. In the past few decades, with the rapid development of computing technology, artificial intelligence (AI) technologies have made remarkable progress and been applied in various fields. Deep learning (DL), as the hottest technology in AI, has gradually become the focus of machine learning research [14]. Deep learning technologies can automatically mine and analyze the data characteristics of labeled data, which are suitable for image segmentation applications. Indeed, the left ventricular NMR image is easy to be segmented for its clear image boundary. However, because of the thin wall of the right ventricle, distinguishing the boundary between the right ventricle inner and outer membranes is more difficult than that of the left ventricle. In addition, right ventricle NMR images are difficult to obtain, and the number of public NMR images is small, which leads to a situation where the research on right ventricle is much less than that of left ventricle. In order to study the right ventricular NMR image segmentation, in this paper, 16,200 NMR images were collected from 128 patients in a hospital of Gansu Province, China. And the ventricular edges of these images were preprocessed and labeled. We try to make full use of the boundary characteristics of ventricular layers and have applied deep learning models to segment ventricle NMR images.

The rest of this paper is organized as follows. Section 2 introduces the related work, which mainly includes traditional methods and deep learning methods for ventricular image segmentation. Section 3 shows the proposed layered method for ventricular image segmentation. Meanwhile, we also propose to develop automatic abnormal systolic function detection system based on the proposed ventricular segmentation model. Section 4 presents the ex- 
perimental results of the proposed method. Finally in Section 5, we conclude this paper.

\section{Related Work}

With the competition tackling the challenges the left ventricular segmentation initiated by Kaggle in 2016 [15], the ventricular segmentation has been widely researched. Researchers have used various methods to segment the ventricle NMR images. However, there are still many difficulties and challenges in ventricular segmentation, especially for right ventricular segmentation. The methods for NMR image segmentation are generally divided into two categories, one type is the traditional segmentation algorithms [18, 28], and another type is the deep learning based methods, which are more and more widely researched in the field of automatic ventricular segmentation and have achieved better and better segmentation results [22]. The difficulty of ventricular image segmentation lies in the complexity of heart structure. In addition, the shape and size of ventricles vary greatly between different individuals. Therefore, it is difficult to identify and judge the inner structure of ventricle.

\subsection{Traditional methods}

The traditional methods for ventricular NMR image segmentation mainly include threshold segmentation method [20], region growing method [27], clustering analysis method [3], level set segmentation method [5] and graph cutting algorithm [8]. Varga-Szemes et al. realized semi-automatic ventricular threshold segmentation algorithm based on the intensity of myocardial signals. Their results showed competitive results than conventional methods. In their experiments, the left ventricle had a large contrast in gray value due to the thickness of myocardial wall, which achieved good segmentation results [24]. Although threshold segmentation methods need small amount of computation, it does not consider spatial characteristics and is sensitive to noise. Hence, the threshold segmentation methods need to be improved [13]. Wang studied traditional ventricular segmentation methods in detail and achieved good results. Ayed et al. proposed a method based on discrete kernel density matching energy function to segment the left ventricle, which is a flexible and high computational performance image segmentation algorithm [1]. However, it only has good result on image segmentation with high contrast, and the global energy function can only achieve local optimum. Based on the above analysis, we can see that the traditional image segmentation methods have their own characteristics and have achieved good results in some professional fields. However, these methods did not perform well in the field of ventricular segmentation, due to the variability of ventricular shape and the large variation of ventricular periphery during cardiac cycle. In addition, most traditional segmentation methods are semi-automatic, which undoubtedly increases the workload of medical staff. 
Therefore, it is urgent to develop accurate and efficient automatic ventricular segmentation methods.

\subsection{Deep learning methods}

Deep learning can automatically learn the high-level features of the potential distribution of samples, and it outperforms many traditional machine learning algorithms in many computer vision fields. Therefore, deep learning has been gradually applied into the field of ventricular segmentation in recent years. Deep learning based ventricular segmentation algorithms mainly include fully convolutional networks (FCN), U-Net $[9,21]$ and Recurrent Neural Network (RNN) [16]. FCN is designed to classify image at pixel level. Bai et al. have proved that deep learning has great potential for image segmentation [2]. Tran et al. trained deep FCN based on transfer learning and tested 22,000 images for ventricular NMR image segmentation [23]. In their results, the average dice metric (DM) metrics of the left and right ventricles are 0.92 and 0.84, and they achieved good result for left ventricular segmentation. In 2015, Ronneberger et al. [21] proposed the U-Net network based on FCN, which was widely used in medical image segmentation. In U-Net, the shallow layers are used to locate pixels, and the deep layers are used to classify the pixels. UNet includes same number of upsampling layers and downsampling layers. In the ventricular segmentation challenge initiated by Kaggle in 2016, Chuckyee achieved DM 0.83 of right ventricle and DM 0.88 of left ventricle by using expanded convolution operation and adding global receptive field, and the segmentation accuracy exceeded FCN [29]. Cong et al. designed a reverse U-Net network with an end-to-end filter. They achieved best segmentation result of DM metric 0.94 in the left ventricular segmentation, based on the medical image computing and computer-assisted intervention (MICCIAI) dataset [6], but they did not test the right ventricle images. Clement et al. designed a new type of deep convolutional neural network (CNN), which could be regarded as an improvement and enhancement of U-Net. It could automatically learn advanced features and low-level features, which obtained a DM metric of 0.94 in the left ventricle and a DM metric of 0.89 in the right ventricle, which was the best segmentation results at present [32]. Fatemeh et al. combined the CNN model with RNN model and achieved accurate results for segmenting ventricular end-diastolic and end-systolic [7]. Indeed, RNN is mainly used in the field of head CT and electrocardiogram, and it is rarely used in the field of ventricular image segmentation. $\mathrm{Hu}$ and Yin et al. utilized machine learning technology to developed an online ventricular segmentation system [30] based on Yin's work during postgraduate [31]. Due to the complex environment of ventricles, deep learning provides a better solution for precise ventricular segmentation by automatically mining high-level features, and the accuracy is higher than the traditional method. Based on the above analysis, this paper try to apply the deep Mask R-CNN model into the ventricular NMR image segmentation 
to solve the low accuracy problem, and provide method to develop automatic medical diagnosis system.

\section{Our proposed method}

This section presents the details of proposed ventricular segmentation method for ventricular image segmentation. The ventricle images include three areas: left ventricle area, right ventricle area and background area. And there is only one instance for each area. The NMR images are low-resolution gray scale with size of $208 \times 174$, and Mask R-CNN is selected as the deep learning segmentation model. We also introduce the method for abnormal ventricular systolic function detection based on the proposed segmentation model at last.

\subsection{Mask R-CNN model}

In 2017, Mask R-CNN was proposed as the enhanced model of Faster R-CNN, by expanding the function of Faster R-CNN target detection, and the network added mask branch to detect the category of each pixel in the target area [11].

\subsubsection{ResNet101 + FPN network}

Generally, deeper neural network model has stronger expressive ability and more complex function mapping relationship. However, deeper structure may lead to the gradient vanishing or gradient explosion problems [19]. Residual neural network (ResNet) solves this problem by transforming the input layer into residual function, which makes the network easier to be optimized. ResNet network uses fast connection method to reduce the negative effect of network depth. Meanwhile, it reduces the number of parameters to speed up the network training, which makes it more suitable for large-scale neural network structure. According to the experimental results of [10], the error rate decreases with the increase of network depth gradually. Considering computing performance, ResNet101 is selected in this paper, which has 101 layers. However, with the increase of the number of layers, the accuracy for recognizing small objects will decrease. Generally, the ventricle area only accounts for $10 \%$ of the whole image, which will undoubtedly get inaccurate results. Therefore, the backbone detection network needs to be redesigned to have the ability to recognize objects with different size. On the other hand, the feature pyramid network (FPN) is designed to solve this problem. On the basis of ResNet network, FPN adds pyramidal multi-level convolution fusion technology, which connects different convolution layers to transfer the location of the bottom layer and the semantic information of the upper layer between these layers. This new method can be used for different sizes object recognition. According to the above analysis, this paper combines ResNet101 network and FPN as the backbone network of ventricular segmentation. 


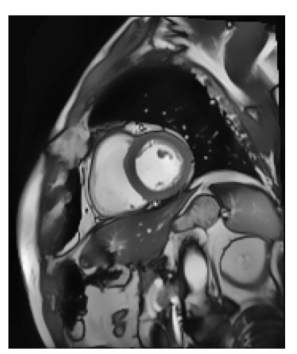

(a)

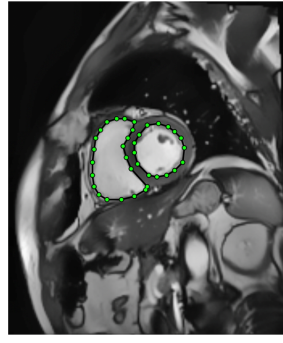

(b)

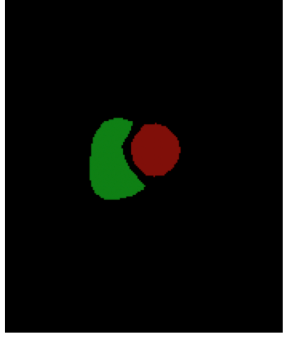

(c)

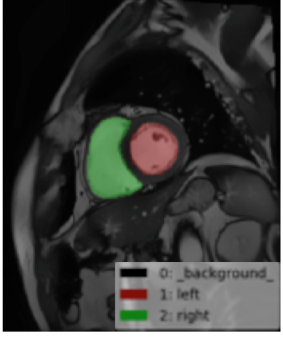

(d)

Fig. 1 The ventricular segmentation images [31]

\subsubsection{Segmentation mask}

Mask file used in image segmentation is a two-dimensional matrix, which is used to label the target area in the image, and it is usually a binary matrix, in which target area is marked as 1 and background area is marked as 0 . In order to generate the mask matrix, we need to generate a matrix with the same size as the image, and all values in the matrix are initialized as 0 . Then, we mark the marginal region of the ventricle, and the values of corresponding positions in the mask matrix are set as 1 . Therefore, by multiplying the original image matrix with the mask matrix, the pixel values in the background region will be 0 , and the target image can be obtained. Fig. 1 (a), (b), (c) and (d) represent the original NMR image, marked image, mask file and the instance segmentation image, respectively. The green area represents the right ventricle and the red area represents the left ventricle.

\subsubsection{Loss function}

In the design of Mask R-CNN, the loss function $L$ consists of three parts, which are classification loss Lcls, boundary loss Lbox and mask loss Lmask, as shown in Eq. (1):

$$
L=L c l s+L b o x+\text { Lmask }
$$

In our experiments, we found that the boundary loss $L b o x$ played an important role in the loss function. The definition of Lbox greatly influences the segmentation model. Therefore, we will tune the position of the boundary box to find the best parameters when training the model.

\subsection{Ventricular NMR images segmentation}

Generally, ventricle NMR images include multi-layer images, and there are many differences in size and details between these layers. Therefore, NMR images are designed to be segmented based on layered method in this paper, which makes the segmentation results more accurate. 


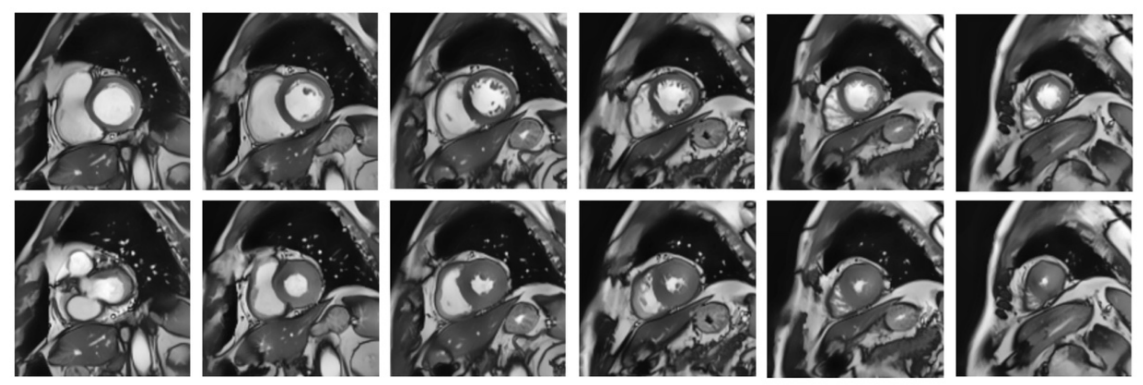

Fig. 2 The six layers ventricle images

Fig. 2 shows the 6 layers NMR images of a patient from tricuspid valve position to apex position (1-6 slices). The upper images show the slices of diastolic images, and the bottom images show the slices of end-systolic images. In reality, the features in different NMR layers are different. For conditions with less marked dataset, using all these images in different layers to train the model will lead to large prediction error. Hence, we collected and marked a large dataset in this paper to separately train segmentation models for different layers.

\subsection{The design of the segmentation models}

In this paper, we trained deep segmentation models for 6 layers NMR images, and the models correspond to the corresponding 6 ventricle slices. The first five layers correspond to the first five models, and the remaining layers are placed in the sixth model because the the remaining layers are closer to the apex, which is similar to the sixth layer.

Fig. 3 shows the overall design of our novel method. Because of the design of layered Mask R-CNN method, NMR image in each layer will be segmented by an independent Mask R-CNN model. The redundant layers will be merged into the sixth layer model for training and prediction. The Mask R-CNN model combines ResNet101 and FPN as the backbone model structure, and the region proposal network (RPN) is used to extract candidate regions by a sliding window. As shown in Fig. 3, the region of interest (ROI) classifier and boundary box regressor are also introduced to fine-tune candidate windows. ROI classifier can be used to select interesting and important areas of segmentation, and boundary box regressor can be used to accelerate the search of ROI by regressor. Therefore, the introduction of these two methods can improve the accuracy of ventricular segmentation. 


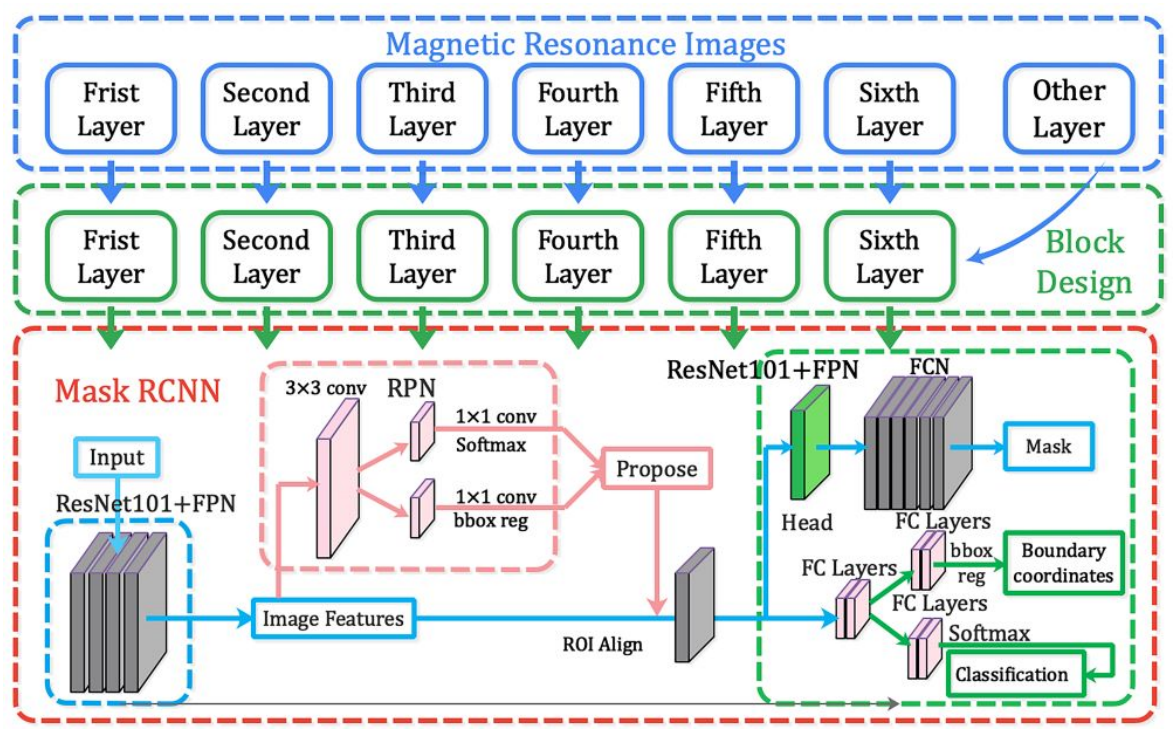

Fig. 3 The design of six-layer ventricular segmentation method

\subsection{Abnormal systolic function detection}

Medically, the health status of the ventricle is usually determined by the end of ventricular diastolic and the end volume of contraction. Although the ventricular size is very different for different people, the amplitude of ventricular contraction is similar, which can be used to analyze the state of ventricular function. In this part, we extract the end of diastolic and shrinklasting images based on the layered ventricular segmentation models. Then, we try to detect ventricular systolic dysfunction by analyzing the ratio of these two types ventricular slice areas.

In Fig. 4, the upper row shows the ventricle images of a healthy person, and the bottom row shows the ventricle images of a patient. It can be seen from the figure that the amplitude of normal healthy person's ventricular contraction is large. While the amplitude is several times decreased for patient images. Meanwhile, the volumes of ventricle images of patients are far greater than that of healthy person. Therefore, the ratio of diastolic to systolic NMR slice area can be used to detect abnormal systolic function.

We used the NMR images including 116 patients with normal ventricular systolic function and 12 patients with decreased ventricular systolic function and dilated heart disease for experiments. Because of the similarity between six layers of the ventricle slice images, we select the second layer to conduct ventricular systolic dysfunction experiments. The second layer performs better in both DM and HD coefficients. Meanwhile, it also has a clear target area in the ventricle images. 

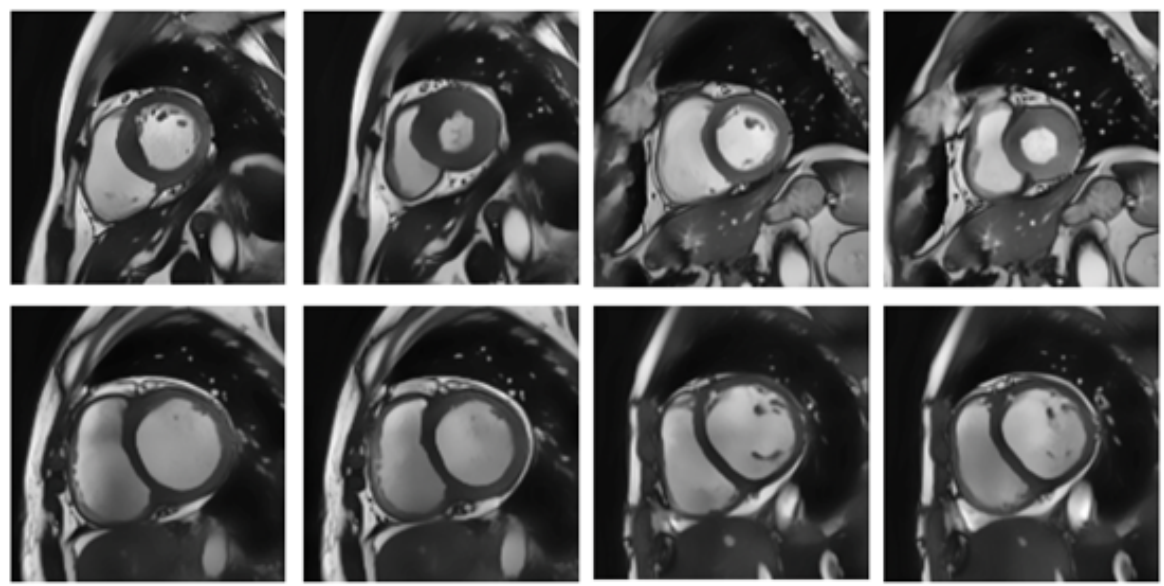

Fig. 4 The second layer ventricular images of healthy people and patient [31]

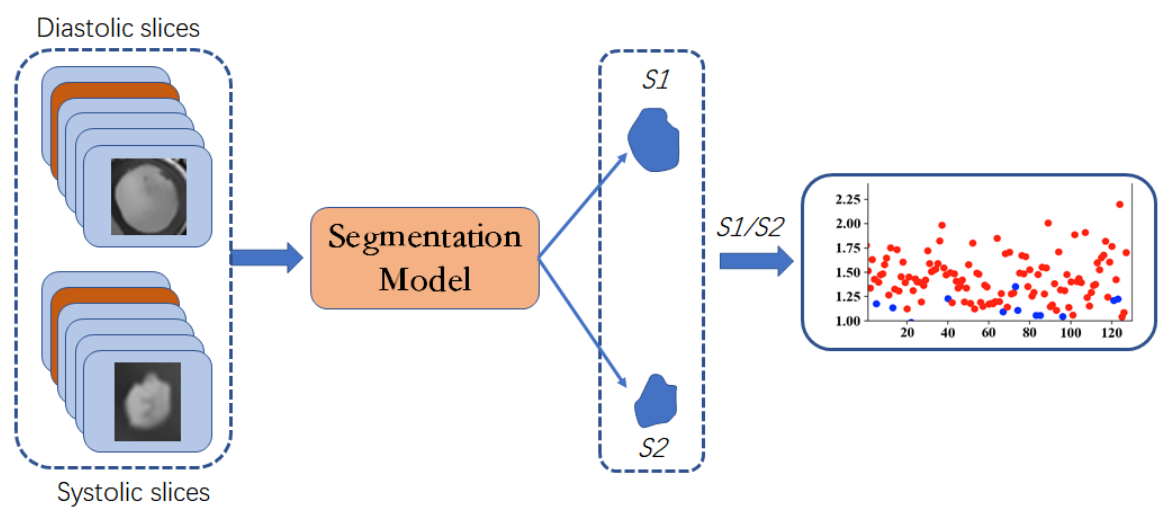

Fig. 5 Abnormal systolic function detection based on segmentation model

As shown in Fig. 5, the diastolic and systolic slice images of the second layer are extracted and inputted into the segmentation model. Next, the ventricular areas are automatically segmented based on proposed segmentation model. Then, we analyze the range of the area ratio of diastolic image to systolic image, which can be used to detect abnormal ventricular systolic function. In this process, the whole segmentation and ratio analysis process is completed by program automatically. Therefore, it can automatically be used as an auxiliary diagnostic tool for doctors.

\section{Experimental Results and Anslysis}

In this section, we show the experiments on the ventricular segmentation. The experiments were carried on Ubuntu 16.04 with a GeForce GTX 1080 GPU. 
The framework of deep learning was Keras 2.2.4, based on Python 3.6.5 and TensorFlow-gpu 1.9.0.

\subsection{Dataset}

There are few public ventricle NMR image datasets available at present. ACDC MICCAI [64] contributed a dataset for ventricular segmentation challenge in 2017. The dataset provides small samples, which are suitable for small-scale models, and it is easy to cause over-fitting problem for large-scale network model such as Mask R-CNN. Therefore, we collected a new ventricle NMR image dataset, which includes data of 128 patients in a hospital in Gansu Province of China. The Siemens Skyra 3.0T MR scanner was used to scan the hearts. The NMR images include 6 to 8 layers, and each layer is $8 \mathrm{~mm}$ thick, including 25 scanned images in one cardiac cycle. The images are in digital imaging and communications in medicine (DICOM) format, and the image resolution is $208 \times 174$. We collected 16,200 ventricle images (including left and right ventricles) under the guidance of professional doctors, and the ventricle edges were marked in these images accurately manually. In our experiments, we selected 13000 images as training samples, and the remaining 3,200 images were used for test.

\subsection{Evaluation metrics}

DM metric and HD metric are often used to evaluate the results of ventricular image segmentation.

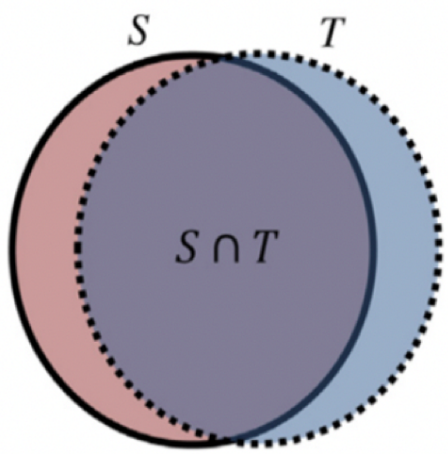

(a)

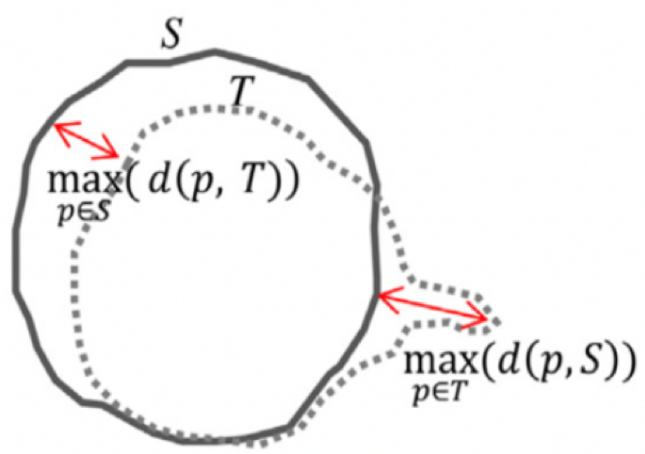

(b)

Fig. 6 The DM and HD evaluation metrics

As shown in Fig. 6 (a), the DM metric is an evaluation method based on similarity measure. $S$ represents the area of the original marked ventricle 
image, and $T$ represents the area of predicted marked image. The DM metric is expressed as Eq. (2).

$$
D M(S, T)=2 \cdot(S \cap T) /(S+T)
$$

HD is also an evaluation method based on similarity measure and it expounds the similarity between point sets. As shown in Fig. 6 (b), $S$ and $T$ represent the boundary of original and predicted masks, respectively. The HD metric is calculated by comparing all points in $T$ and all points in $S$ to get the farthest metrics that cover all directions and angles in $S$ and $T$. The formula of $\mathrm{HD}$ is as Eq. (3).

$$
\begin{array}{r}
d(p, T)=\min _{p^{\prime} \in T}\left\|p-p^{\prime}\right\| \\
H a u s d o r f f(S, T)=\max \left\{\max _{p \in T} d(p, S), \max _{p \in S} d(p, T)\right\}
\end{array}
$$

In this paper, the experimental results are evaluated by DM and HD metrics, which are also used to compare with other methods in existing studies.

\subsection{Model training}

Transfer learning is used for the pre-training of the segmentation models, which is helpful for the rapid convergence of deep models. In our experiments, we use models trained by the $\mathrm{COCO}$ dataset to initialize the segmentation models. In fact, the COCO dataset has more than 120,000 categories [25]. So the deep learning models trained by the COCO dataset can be easily transferred to new application scenarios with recognition ability. Meanwhile, the models can converge quickly in training and achieve good classification results after initialization through transfer learning, which has been verified on the Mask R-CNN model [12].

There are also many other important parameters in Mask R-CNN affecting the results of the model. Therefore, we need to combine the experimental results and analysis to find best models.

Fig. 7 shows the loss curves for training six layer models. SAX1-SAX6 denote segmentation models for first short-axis slice (SAX) to sixth SAX. SAX1 is near the tricuspid layer and SAX6 is near the apical layer. As shown in Eq. (1), loss function mainly includes classification loss (class_loss), boundary box regression loss (bbox_loss) and mask loss (mask_loss). From Fig. 7 we can observe that the loss function declines fastest in the first five epochs and finally oscillates in a small loss range. The classification loss is smallest, which indicates that the classification is accurate. The trend of the boundary box loss and mask loss is similar. The training processes of SAX1 and SAX2 are more smooth, and the first two layers can achieve better results through fewer training steps. The reason behind is that the inner boundaries of the first two layers are clearest. The training processes of the last two layers are very unstable, and all losses are higher than those of other layers. The reason is that 


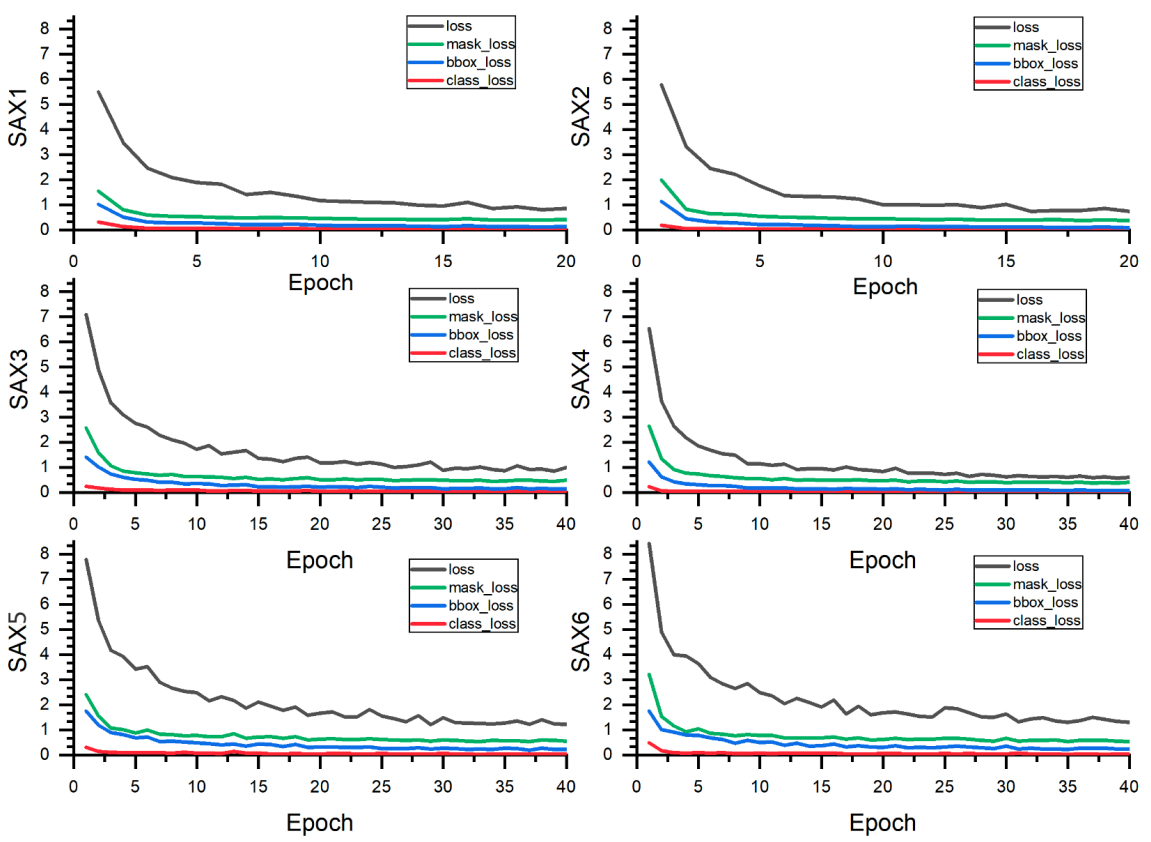

Fig. 7 The loss curves of segmentation models of six layers

Table 1 The evaluation results of the deep models for segmenting SAX1-SAX6 of right ventricle (RV) and left ventricle (LV) NMR images.

\begin{tabular}{lcccc}
\hline & \multicolumn{2}{c}{ DM } & \multicolumn{3}{c}{ HD } \\
\cline { 2 - 5 } & RV & LV & RV & LV \\
\hline SAX1 & 0.9183 & 0.9311 & 6.75 & 4.75 \\
SAX2 & 0.9140 & 0.9226 & 6.86 & 4.92 \\
SAX3 & 0.8834 & 0.9279 & 8.12 & 5.03 \\
SAX4 & 0.8683 & 0.9275 & 8.44 & 4.86 \\
SAX5 & 0.8776 & 0.9228 & 6.26 & 4.77 \\
SAX6 & 0.8819 & 0.9176 & 5.83 & 4.32 \\
Average & 0.8906 & 0.9249 & 7.04 & 4.78 \\
\hline
\end{tabular}

the target area of the end-systolic in the last two layers images is generally too small, and it is difficult for the boundary frame to detect them, which results in large losses and fluctuations.

\subsection{Segmentation results analysis}

Table 1 shows the DM and HD metrics of right ventricular segmentation results from first layer to sixth layer. We can see that the average DM is 0.8906 and average $\mathrm{HD}$ is $7.04 \mathrm{~mm}$. Also, we can see that the DM metrics of the first 
two layers are higher than 0.91 and they show better performance. The third layer images have a large target area, but the inner and outer membrane boundary is more difficult to identify than the first two layers, which leads to a slight drop in accuracy. The segmentation results of the remaining layers in the right ventricle are similar, which are nearby 0.87 . The inner and outer membranes of the last two layers almost coincide during ventricle contraction, and there is no need to judge the inner and outer membranes. Therefore, the segmentation results of last two layers improve a little than the middle layers. Table 1 presents the results of DM and HD in all layers of left ventricle. The average DM is 0.92 and average $\mathrm{HD}$ is $4.78 \mathrm{~mm}$. Because the left ventricle is more regular and the ventricle edge are clearer, the DM of left ventricle is generally above 0.91 , which is better than that of right ventricle. The HD metric of left ventricle is generally lower than that of right ventricle, because the left ventricle is easier to be distinguished than right ventricle.
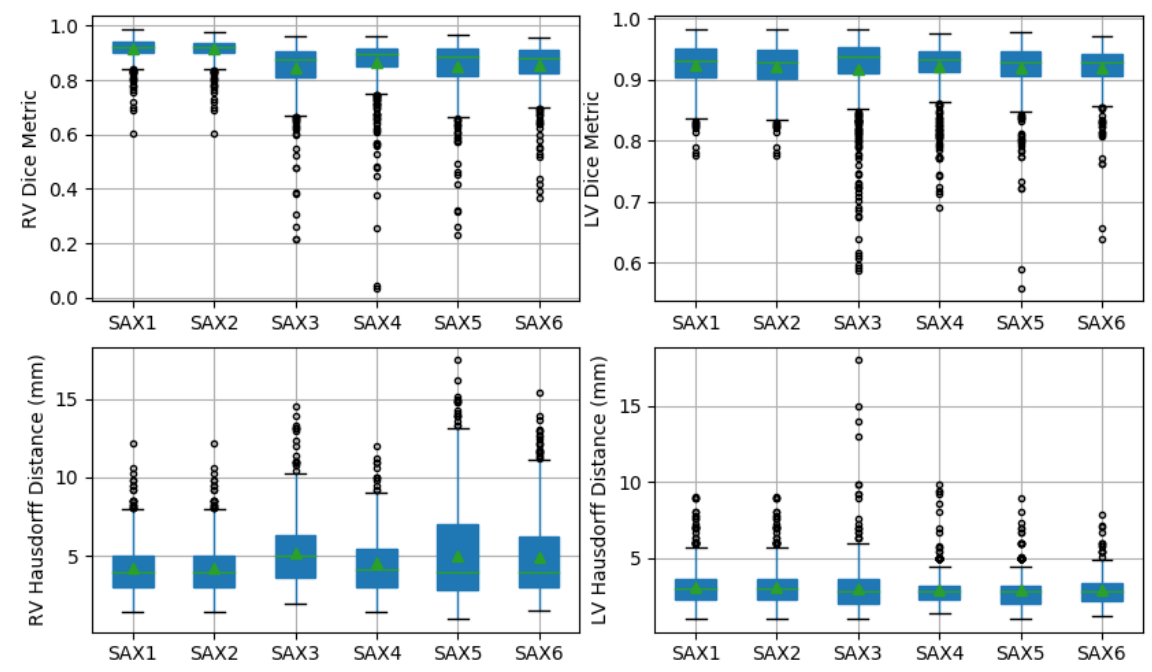

Fig. 8 The distribution of DM and HD in six layers

Fig. 8 shows the distribution of DM and HD in each layer for left and right ventricular segmentation. We can see that the number of abnormal points of DM and HD in the left ventricle is less than that in the right ventricle. By checking the NMR images, we found that the abnormal points usually appeared in the images of patients who had small ventricle area or arrhythmia, which led to low DM and high HD.

Fig. 9, (a)-(f) show the ventricular segmentation results from the first layer to the sixth layer. There are two groups of ventricular segmentation images for different patients in one layer. The left image in each group is the mask file marked manually, and the right image is the predicted segmentation image. The blue area represents right ventricle, and the red area denotes left ventricle. 
(a)
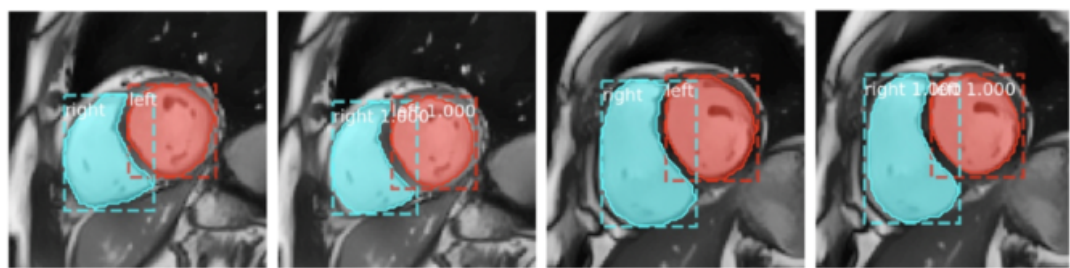

(b)
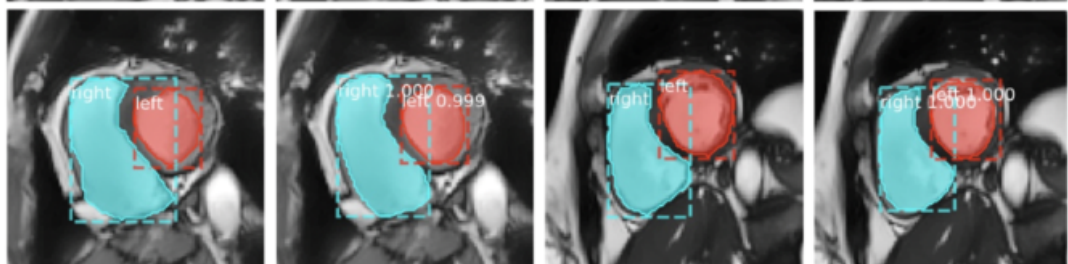

(c)
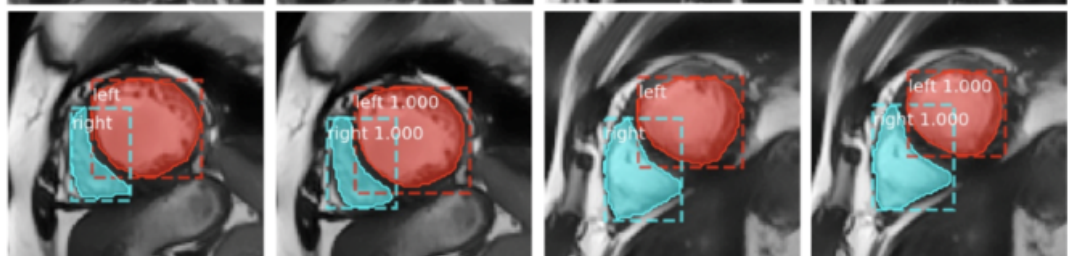

(d)
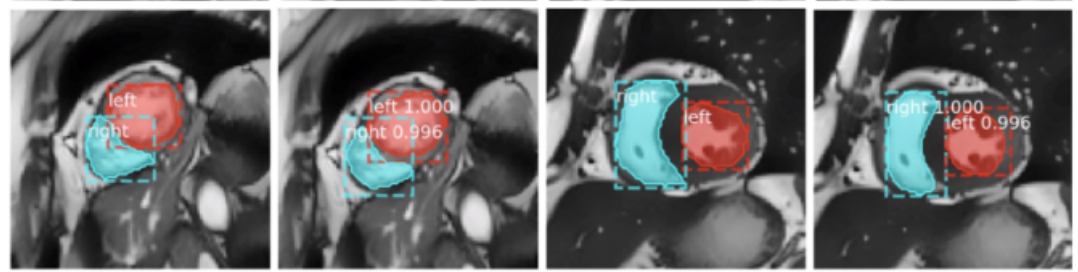

(e)
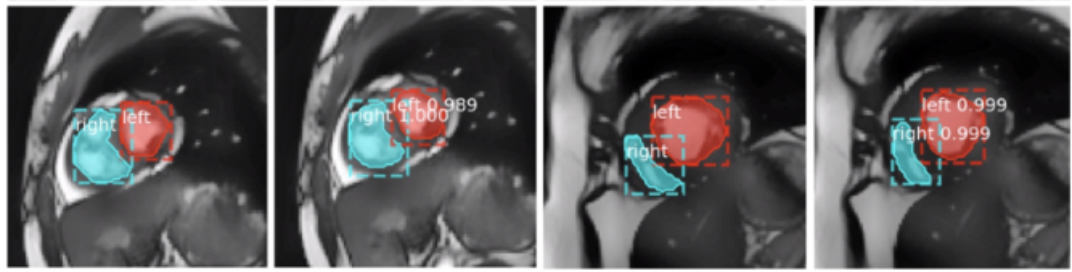

(f)
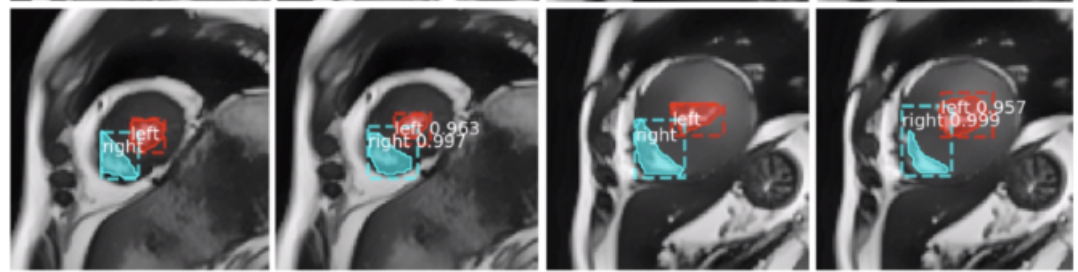

Fig. 9 The six layers ventricular image segmentation results 
The numbers in the predicted rectangle boxes show the DM scores. We can observe that the predicted segmentation images are very smooth, and the accuracies of left and right ventricle edge prediction are high.

From Fig. 9 (a) and (b) we can see that the detection results of left and right ventricles in the first two layers are good, because the target area is easier to identify than other layers. The average HD is low and stable, which proves that the layered Mask R-CNN model is capable for ventricular edge segmentation. Fig. 9 (c) and (d) shows the segmentation results of the third and fourth layers. We can observe that the target area becomes smaller and there are some interference factors such as trabecular muscles. On the other hand, the predicted image is relatively accurate, but a little worse than that of the first two layers. Fig. 9 (e) and (f) show the segmentation results of small ventricle images in the fifth and sixth layers. We can see that the forecasting errors of RPN boundary box and ventricle edge get larger. However, benefiting from the boundary box of Mask R-CNN, the segmentation results are acceptable.

\subsection{Ventricular segmentation results comparision}

In this section, the experimental results of proposed method and the state of the art methods are compared based on same dataset, and the advantages and disadvantages of these algorithms in ventricular segmentation are summarized. The comparison results are shown in Table 2.

Table 2 The ventricular segmentation results of proposed method and other methods for right ventricle $(\mathrm{RV})$ and left ventricle $(\mathrm{LV})$.

\begin{tabular}{lcccc}
\hline & \multicolumn{2}{c}{ DM } & \multicolumn{2}{c}{ HD } \\
\cline { 2 - 5 } & RV & LV & 8.98 & LV \\
\hline$[26]$ & 0.87 & - & 8.86 & - \\
{$[23]$} & 0.84 & 0.92 & 16.78 & 5.79 \\
{$[32]$} & 0.94 & $\mathbf{6 . 1 1}$ & 4.68 \\
SAX1 & 0.88 & 0.9316 & $\mathbf{6 . 2 6}$ & 4.70 \\
SAX2 & $\mathbf{0 . 9 2 7 9}$ & 0.9272 & $\mathbf{7 . 3 9}$ & 5.01 \\
SAX3 & $\mathbf{0 . 9 2 2 5}$ & 0.9145 & $\mathbf{8 . 2 2}$ & 5.28 \\
SAX4 & $\mathbf{0 . 8 8 8 0}$ & 0.9024 & $\mathbf{8 . 3 4}$ & 6.31 \\
SAX5 & 0.8655 & 0.8508 & $\mathbf{8 . 6 4}$ & 6.75 \\
SAX6 & 0.8359 & 0.8412 &
\end{tabular}

The boldfaces represent better results than other methods, and "-" denotes lacking results.

Among all the other methods, [26] shows the segmentation results of traditional methods. We can see that the traditional method has no advantage over our method in segmentation results. [32] performs best in DM for left ventricle, but it performs poorly in HD for right ventricle. Actually, [32] is an extension of U-Net model, and it is a type of best method in the field of 
ventricular segmentation at present. [23] performs well in HD metric, but it performs poorly in DM metric. Our proposed method performs best in DM for the right ventricular segmentation in the first three layers, and the results of first two layers are the most prominent, which are equal to results in [32]. Anyhow, the average accuracy of left ventricle is lower than [32].

In [32], the lowest HD metrics of right ventricle and left ventricle are 13.03 $\mathrm{mm}$ and $4.16 \mathrm{~mm}$. Compared back to back, the average HD of right ventricle and left ventricle are $7.49 \mathrm{~mm}$ and $5.46 \mathrm{~mm}$ in our results. Although the proposed method obtains similar results as [32] in left ventricular segmentation, it outperforms [32] in right ventricular segmentation. It indicates that the proposed method is more accurate in locating the target area, and it performs better than [32] in ventricle detection.

In summary, the proposed layered Mask R-CNN method achieves good segmentation results in both DM and HD metrics, especially for right ventricle segmentation, which has rarely been achieved before.

4.6 Analysis of abnormal systolic function detection
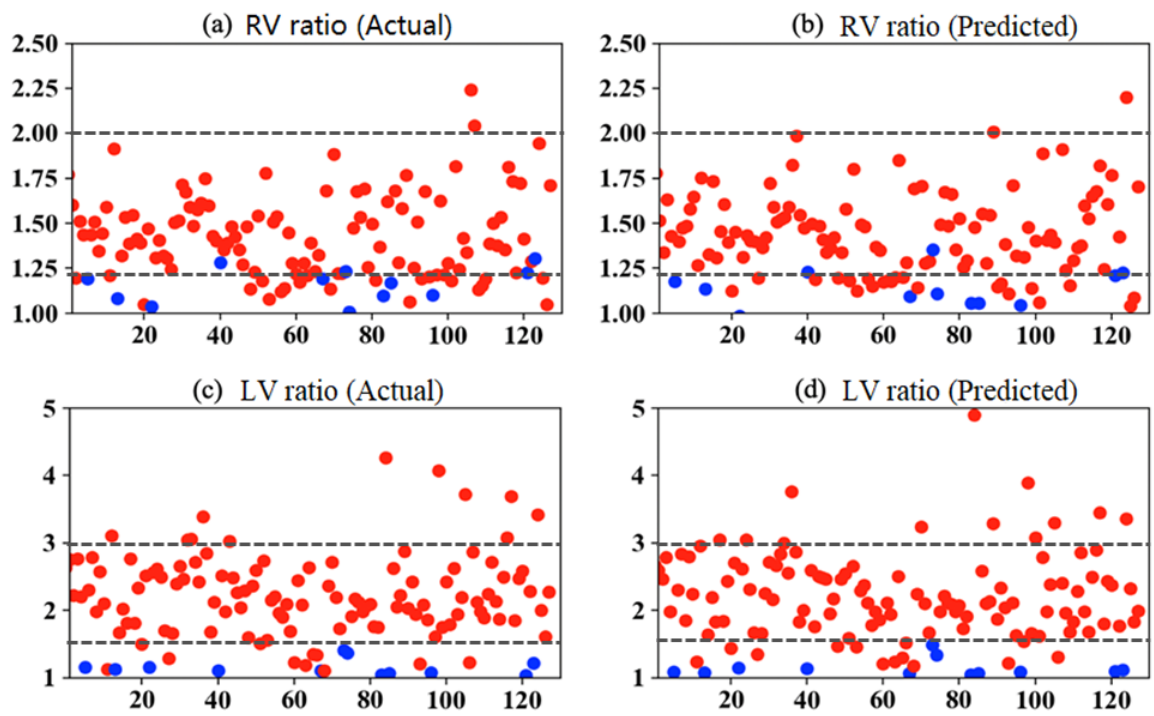

Fig. 10 The area ratio distribution of healthy people and patients

The area ratios of diastolic to systolic are given in Fig. 10. Figure (a) and (c) show the actual ratio distributions based on manually labeled right and left ventricular images, and figure (b) and (d) show the predicted ratio distributions based on segmented right and left ventricular images by the segmentation model. The ordinate denotes the ratios, and the horizontal ordinate shows the patient number. 
We can observe that the ratios of patients are significantly lower than that of healthy people. Meanwhile, for the right ventricle, the ratios of healthy people is mainly between 1.2 and 2.0. And it ranges between 1.5 and 3.5 for the left ventricles of healthy people. In fact, the left and right ventricular contractions are usually synchronous. If the right ventricular ratio is low, then the person's left ventricular ratio is also low. Based on this synchronization, we can take the one left or right ventricular contraction ratio as the detection parameter. This rule is also preserved in the automatic ventricular segmentation images predicted by segmentation model, as shown in figure (b) and (d). It indicates that the proposed ventricular segmentation model can not only be used for ventricular segmentation, but also can be used for automated ventricular systolic disease detection, which helps to develop auxiliary medical diagnosis system.

\section{Conclusions}

The diagnosis of CVDs mainly relies on MRI technology, and the traditional analysis of MRI images needs help from professional doctors, which is timeconsuming, laborious and easy to cause misdiagnosis. This paper proposed an automatic ventricular segmentation method to assist the diagnosis of CVDs. We collected and marked a dataset including 16,200 NMR images. Based on the dataset, a layered Mask R-CNN segmentation method was proposed to automaticlly detect the ventricle edge in the NMR images. The experimental results show that the mean DM metrics of right and left ventricles are 0.89 and 0.92 , and the mean HD metrics of right and left ventricles are $7.03 \mathrm{~mm}$ and $4.78 \mathrm{~mm}$. Compared with earlier popular methods, our proposed method is more accurate for right ventricular segmentation. It also shows strong stability and robustness for small target areas. We also experimentally confirmed this automatic ventricular NMR image processing method can be used for automated ventricular systolic disease detection.

Future studies may focus on the improvement of model accuracy, and extended applications such as the calculation of ventricular volume, ejection fraction calculation and more heart disease detection.

Acknowledgements This work was partially supported by National Natural Science Foundation of China under Grant No. 61402210 and 61872079, The Fundamental Research Funds for the Central Universities under Grant No. lzujbky-2019-kb51 and lzujbky-2018-k12, Ministry of Education - China Mobile Research Foundation under Grant No. MCM20170206, Major National Project of High Resolution Earth Observation System under Grant No. 30Y20A34-9010-15/17, State Grid Corporation Science and Technology Project under Grant No. SGGSKY00FJJS1800403 and No.522722160071, Program for New Century Excellent Talents in University under Grant No. NCET-12-0250, and Double first class FundingInternational Cooperation and Exchange Program under Grant No. 227000-560001, and Strategic Priority Research Program of the Chinese Academy of Sciences with Grant No. XDA03030100. Google Research Awards and Google Faculty Award. We appreciate coauthor Mr. Hang Yin for the contribution of this paper is based on his master thesis [31] and co-authored conference paper [30] and his hard work during postgraduate. Jun Shen's 
work was supported by international expert exchange programs and UOW's UGPN RCF 2018-2019.

\section{References}

1. Ayed IB, Punithakumar K, Li S, Islam A, Chong J (2009) Left ventricle segmentation via graph cut distribution matching. International Conference on Medical Image Computing and Computer-Assisted Intervention pp 901-909

2. Bai W, Shi W, Ledig C, Rueckert D (2015) Multi-atlas segmentation with augmented features for cardiac mr images. Medical Image Analysis 19(1):98-109

3. Ballaarabe S, Gao X, Ginhac D, Brost V, Yang F (2017) Architecturedriven level set optimization: From clustering to subpixel image segmentation. IEEE Transactions on Cybernetics 46(12):3181-3194

4. Brown AF, Liang LJ, Vassar SD, Escarce JJ, Merkin SS, Cheng E, Richards A, Seeman T, Jr LW (2018) Trends in racial/ethnic and nativity disparities in cardiovascular health among adults without prevalent cardiovascular disease in the united states, 1988 to 2014. Annals of Internal Medicine 168(8):541-549

5. Chunming L, Rui H, Zhaohua D, J Chris G, Metaxas DN, Gore JC (2011) A level set method for image segmentation in the presence of intensity inhomogeneities with application to mri. IEEE Transactions on Image Processing 20(7):2007-2016

6. Cong, Zhang (2018) Invert unet dnn segmentation model for mri cardiac left ventricle segmentation. The Journal of Engineering 16:1463-1467

7. Dezaki FT, Liao Z, Luong C, Girgis H, Tsang T (2018) Cardiac phase detection in echocardiograms with densely gated recurrent neural networks and global extrema loss. IEEE Transactions on Medical Imaging 1(99):1821-1832

8. Grosgeorge D, Petitjean C, Caudron J, Fares J, Dacher JN (2011) Automatic cardiac ventricle segmentation in mr images: A validation study. International Journal of Computer Assisted Radiology \& Surgery 6(5):573 581

9. Han Y, Ye JC (2018) Framing u-net via deep convolutional framelets: Application to sparse-view ct. IEEE Transactions on Medical Imaging 37(6):1418-1429

10. He K, Zhang X, Ren S, Jian S (2016) Deep residual learning for image recognition. In: IEEE Conference on Computer Vision \& Pattern Recognition, pp 770-778

11. He K, Gkioxari G, Dollar P, Girshick R (2017) Mask r-cnn. In: 2017 IEEE International Conference on Computer Vision (ICCV), pp 2961-2969

12. Hu R, Dollár P, He K, Darrell T, Girshick R (2018) Learning to segment every thing. Proceedings of the IEEE Conference on Computer Vision and Pattern Recognition pp 4233-4241 
13. Juan S, Cheng HD, Yuxuan W (2012) Completely automated segmentation approach for breast ultrasound images using multiple-domain features. Ultrasound in Medicine \& Biology 38(2):262-275

14. Lecun Y, Bengio Y, Hinton G (2015) Deep learning. Nature 521(7553):436

15. Liao F, Xi C, Hu X, Song S (2017) Estimation of the volume of the left ventricle from mri images using deep neural networks. IEEE Transactions on Cybernetics PP(99):1-10

16. Liu F, Chen Z, Wang J (2018) Video image target monitoring based on rnn-lstm. Multimedia Tools \& Applications 78:4527-4544

17. Pan G, Zhuo X, Si S, Jin D (2017) Arrhythmia classification based on wavelet transformation and random forests. Multimedia Tools \& Applications $77(1): 1-18$

18. Petitjean C, Zuluaga MA, Bai W, Dacher JN, Grosgeorge D, Caudron J, Su R, Ayed IB, Cardoso MJ, Chen HC (2015) Right ventricle segmentation from cardiac mri: A collation study. Medical Image Analysis 19(1):187-202

19. Ramaswamy A, Bhatnagar S (2018) Analysis of gradient descent methods with non-diminishing bounded errors. IEEE Transactions on Automatic Control 63(5):1465-1471

20. Ringenberg J, Deo M, Devabhaktuni V, Berenfeld O, Boyers P, Gold J (2014) Fast, accurate, and fully automatic segmentation of the right ventricle in short-axis cardiac mri. Computerized Medical Imaging \& Graphics 38(3):190-201

21. Ronneberger O, Fischer P, Brox T (2015) U-net: Convolutional networks for biomedical image segmentation. International Conference on Medical image computing and computer-assisted intervention pp 234-241

22. Tan LK, Liew YM, Lim E, Mclaughlin RA (2017) Convolutional neural network regression for short-axis left ventricle segmentation in cardiac cine mr sequences. Medical Image Analysis 39:78-86

23. Tran PV (2016) A fully convolutional neural network for cardiac segmentation in short-axis mri. arxiv DOI https://arxiv.org/abs/1604. 00494

24. Varga-Szemes A, Cannao PM, Muscogiuri G, Renker M, Cecco CND, Giri S, Piccini D, Steinberg DH, Schoepf JU (2015) Non-contrast 3d radial and qiss mra for transcatheter aortic valve replacement planning. Journal of Cardiovascular Magnetic Resonance 17(1):1-2

25. Vinyals O, Toshev A, Bengio S, Erhan D (2017) Show and tell: Lessons learned from the 2015 mscoco image captioning challenge. IEEE Transactions on Pattern Analysis and Machine Intelligence 39(4):652-663

26. Wang K (2017) right ventricle segmentation based on cardiac magnetic resonance images. Master thesis, Lanzhou University

27. Wang L, Pei M, Codella NC, Kochar M, Weinsaft JW, Li J, Prince MR, Wang Y (2016) Left ventricle: Fully automated segmentation based on spatiotemporal continuity and myocardium information in cine cardiac magnetic resonance imaging (lv-fast). Biomed Research International 2015:1-9

28. Wu HS, Wang D, Shi L, Yu CM (2013) Automatic segmentation of left ventricle in $3 \mathrm{~d}$ echocardiography using a level set approach. International Journal of Cardiology 164(2):S12-S13 
29. Yee $\mathrm{CH}$ (2018) Cardiac mri segmentation. URL https://chuckyee.github.io/cardiac-segmentation/

30. Yilin H, Hang Y, Binbin Y, Yunshan C, Xing Z, Rui Z, Qingquan L, Mingsong (2019) Online ventricular segmentation system based on machine learning. In: 2019 IEEE Intl Conf on Dependable, Autonomic and Secure Computing, IEEE, pp 309-314

31. Yin H (2019) Research and application of ventricular segmentation using nuclear magnetic resonance imaging based on deep learning. Master thesis, Lanzhou University

32. Zotti C, Luo Z, Lalande A, Humbert O, Jodoin PM (2017) Novel deep convolution neural network applied to mri cardiac segmentation. arxiv DOI https://arxiv.org/abs/1705. 08943v1 